\title{
Percolation and Cluster Distribution. II. Layers, Variable-Range Interactions, and Exciton Cluster Model
}

\author{
J. Hoshen, ${ }^{1}$ R. Kopelman, ${ }^{1}$ and E. M. Monberg ${ }^{1}$ \\ Received December 8, 1976; revised May 23, 1978
}

\begin{abstract}
Monte Carlo simulations for the site percolation problem are presented for lattices up to $64 \times 10^{6}$ sites. We investigate for the square lattice the variablerange percolation problem, where distinct trends with bond-length are found for the critical concentrations and for the critical exponents $\beta$ and $\gamma$. We also investigate the layer problem for stacks of square lattices added to approach a simple cubic lattice, yielding critical concentrations as a functional of layer number as well as the correlation length exponent $\nu$. We also show that the exciton migration probability for a common type of ternary lattice system can be described by a cluster model and actually provides a cluster generating function.
\end{abstract}

KEY WORDS : Percolation; critical exponents; cluster model; exciton migration; critical concentration.

\section{INTRODUCTION}

In a recent paper ${ }^{(1)}(\mathrm{I})$ we introduced a new method to evaluate percolation probabilities and critical percolation concentrations, where we utilized a cluster multiple labeling technique (CMLT) to determine cluster size distributions in a simulated lattice. In this paper we focus our attention on some results involving the site problem for a number of lattice topologies.

Typically, data on the lattice percolation problem are given for the nearest neighbor or next nearest neighbor sites. ${ }^{(2)}$ However, long-range interactions may play a significant role, when quantum mechanical effects ${ }^{(3-5)}$ such as tunneling are encountered. Tunneling of triplet excitons can account for the low percolation concentration threshold detected in substitutionally disordered molecular crystals. ${ }^{(3)}$ Similarly, electrical conductivity of lightly doped semiconductors has been viewed as a long-range percolation process. ${ }^{(4,5)}$

Supported by NSF Grant DMR76-07832.

${ }^{1}$ Department of Chemistry, University of Michigan, Ann Arbor, Michigan. 
It has been demonstrated by Skal et al. ${ }^{(4)}$ that long-range correlations effectively reduce the dependence of the critical percolation concentration on the structural characteristics of the system in question. ${ }^{(5)}$ Hence, percolation in systems with long-range site-site interactions ${ }^{(5)}$ can be described in terms of continuous percolation models ${ }^{(6)}$ (and vice versa). We introduce some basic definitions for two- and three-dimensional systems, but mainly concentrate on results for the two-dimensional (square lattice) topology: critical concentrations, percolation probabilities, and critical exponents. While the critical concentration behavior appears to merge smoothly into a "classical" behavior as the interaction range increases, this is definitely not the case for the critical exponents $(\beta, \gamma)$. The latter essentially are unchanged from the values found for the shortest range topology (square lattice with coordination number 4).

Critical percolation phenomena are characteristic of two- and threedimensional lattices. An interesting question in this context is that of percolation in systems consisting of thin layers. Actually, thin-layer lattices provide an interesting intermediate case between two- and three-dimensional systems. Certain molecular aggregates in biological systems as well as some adsorbed layers and coatings formed by vapor deposition may provide suitable examples for percolation in thin layers. This problem has already been discussed for magnets by Binder, ${ }^{(7)}$ who also derived the critical exponents $\left(\lambda=\nu^{-1}\right)$ related to the finite size scaling theory. ${ }^{(8,9)}$ More work regarding the correlation length exponent $\nu$ and finite-size scaling theory has been done very recently by Levinshtein $e t$ al. ${ }^{(10)}$ and by Sur et al. ${ }^{(11)}$ We give here our results, using simulations on larger lattices and a somewhat different method of data analysis.

We also give some Monte Carlo numerical results on the critical concentration and critical exponents $\beta$ and $\gamma$ of simple two-dimensional lattices (square and triangular), together with some preliminary results on the ratio of "cluster mean sizes" above and below the critical concentration. These results are compared to literature values and discussed accordingly. For the sake of interested experimentalists, we give also some simple graphical presentations of the percolation probability and average cluster size. We also give here a brief summary of "exciton percolation" theory ${ }^{(12)}$ and its relation to lattice percolation and to cluster models in magnetism. This gives some new insight into cluster generating functions, in terms of specially defined ternary ("polychromatic") systems.

\section{METHOD OF COMPUTATION}

A crystal is simulated for the site problem by having each site occupied with a probability $C$ (or unoccupied with a probability $1-C$ ). Here $C$ 
denotes the concentration of one component in a binary random crystal. In the Monte Carlo simulation, sites are represented by vectors $\mathbf{S}$. The occupation of sites is determined by random numbers $X_{i}$ generated in the range $0<X_{i}<1$. A site $i$ is occupied if $X_{i} \leqslant C$. The site is not occupied if $C>X_{i}$. The vector element $S_{i}$ is set to -1 if site $i$ is occupied. Otherwise it is set to 0 . The next step is to determine the cluster size distribution, i.e., the number of clusters of a given size. The determination of the cluster size distribution is achieved by applying the CMLT, ${ }^{(1)}$ which assigns a set of natural numbers

$$
\left\{m_{1}^{\alpha}, m_{2}{ }^{\alpha}, \ldots, m_{s}^{\alpha}, \ldots, m_{t}^{\alpha}\right\}
$$

to label sites belonging to each cluster in the lattice, where $\alpha$ denotes the cluster in question and $m_{s}{ }^{\alpha}$ is the smallest number of the set in Eq. (1) and is defined as the proper cluster label of the $\alpha$ cluster. The labels of Eq. (1) are interrelated by a set of integers,

$$
\left\{\bar{N}\left(m_{1}{ }^{\alpha}\right), \bar{N}\left(m_{2}^{\alpha}\right), \ldots, \bar{N}\left(m_{s}^{\alpha}\right), \ldots, \bar{N}\left(m_{t}^{\alpha}\right), \ldots\right\}
$$

of which only $\bar{N}\left(m_{s}^{\alpha}\right)$ is a positive number and denotes the $\alpha$ cluster size, i.e., the number of sites belonging to the cluster. The labels $m_{t}^{\alpha}$ are related to $m_{s}^{\alpha}$ by

$$
m_{s}^{\alpha}=-\bar{N}\left(-\bar{N}\left(\cdots \bar{N}\left(-\bar{N}\left(m_{t}^{\alpha}\right)\right) \cdots\right)\right)
$$

Hence, the cluster size distribution can be determined from $\bar{N}\left(m_{s}^{\alpha}\right)$ after the simulated lattice is scanned and labeled. The critical percolation concentration $C_{c}{ }^{s}$ is determined from the maximum of the reduced average cluster size $I_{\mathrm{av}}^{\prime}$ function ${ }^{(1)}$ defined by

$$
I_{\mathrm{av}}^{\prime}(C)=\left(\sum_{m=1}^{m_{\max }} i_{m} m^{2}\right) / G-m_{\max }^{2} / G
$$

where $m$ is the cluster size, $m_{\max }$ is the size of the largest cluster, $i_{m}$ is the frequency of occurrence of a cluster of size $m$, and $G=C N$ is the total number of occupied sites in the simulated lattice. The estimated probability $P_{m}$ of locating any cluster of size $m$ is

$$
P_{m}=m i_{m} / G
$$

The probability that any given guest site is a member of the largest cluster is simply

$$
P_{\max }=m_{\max } / G
$$


The relation of the percolation probability $\bar{P}_{\infty}(C)$ to $P_{\max }$ is discussed in Ref. 1 and also in Section 3. We note that our above criterion for finding the critical concentration is an integral and basic part of the results described below.

\section{THE REDUCED AVERAGE CLUSTER SIZE FUNCTION}

In Fig. 1, the second moment for the cluster size distribution

$$
I_{\text {av }}=\sum_{m=1}^{m_{\max }} i_{m} m^{2} / G
$$

is shown for a $200 \times 200$ square lattice. The function $I_{\mathrm{av}}$ rises asymptotically at the percolation thresheld $C_{c}{ }^{s}$. Although it is apparent from Fig. 1 that $I_{\mathrm{av}}$ does not provide an accurate measure for $C_{c}{ }_{c}^{s}$, it does indicate the percolation threshold region. We shall see in the following figures that $I_{\mathrm{av}}^{\prime}$ of Eq. (4) gives a sharper definition for $C_{c}{ }^{s}$. We note that Fig. 1 is mainly for illustration.

In Fig. 2, $I_{\mathrm{av}}^{\prime}$ vs. guest concentration is plotted for four different topologies. The effect of removing the contribution of the largest cluster from the averaging summation [see Eq. (4)] is insignificant below $C_{c}{ }_{c}^{s}$, as can be seen by comparing the square lattice results in Fig. 2 with those in Fig. 1.

At concentrations just below $C_{c}^{s}$, there are several large clusters and the removal of the largest one does not significantly alter the $I_{\mathrm{av}}^{\prime}$ curve, compared

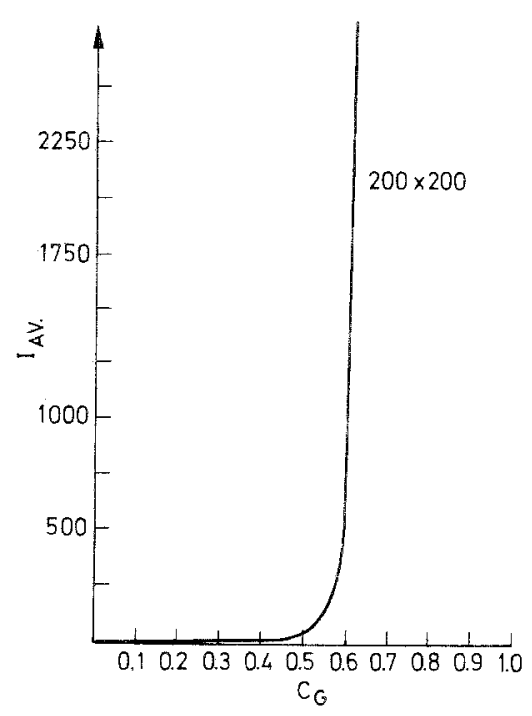

Fig. 1. Average cluster size $I_{\mathrm{av}}$ vs. occupied site (guest) concentration $C_{g}$ in a $200 \times 200$ square lattice; see Eq. (6). Notice that the sharp rise of the curve roughly gives the percolation concentration $C_{\varepsilon}{ }^{s}$. 


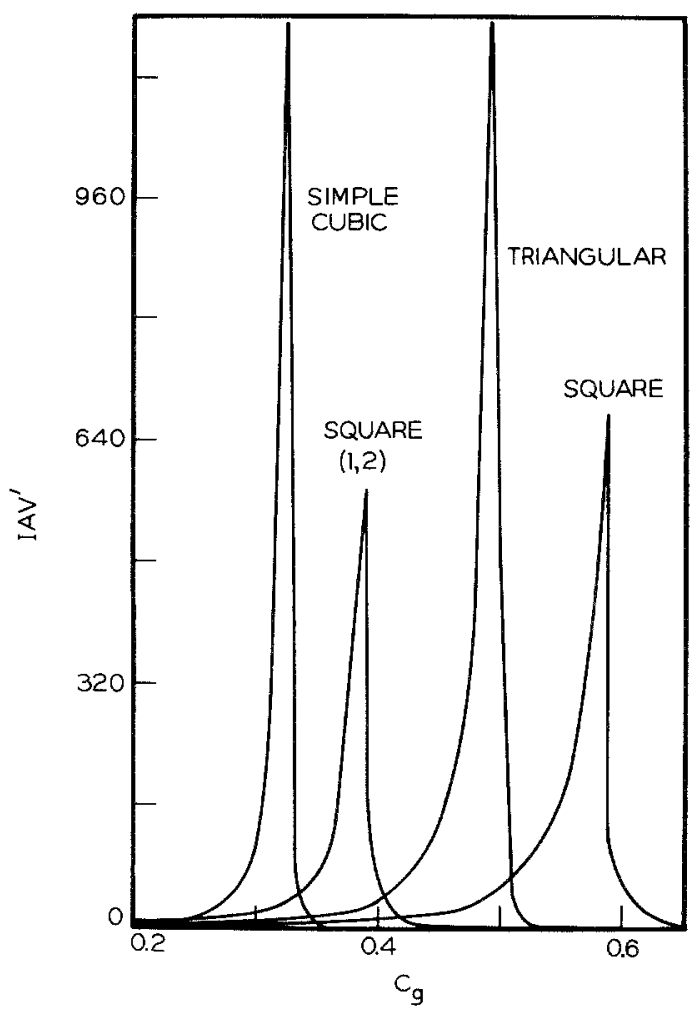

Fig. 2. The reduced average cluster size [Eq. (4)] vs. occupied site (guest) concentration for square, triangular, square $(1,2)$, and simple cubic lattices. The sharp maxima give the respective critical percolation concentrations $C_{c}{ }^{s}$. The three two-dimensional topologies refer to a lattice of $200 \times 200$ sites, while the simple cubic lattice has $100 \times 100 \times 30$ sites (see Fig. 8). The peak heights are a result of statistical fluctuations. (See text.)

to $I_{a \mathrm{v}}$. Only at the point where these large clusters coalesce into one maxicluster does the value of $I_{\mathrm{av}}^{\prime}$ drop precipitously. ${ }^{(1)}$ For any type of topology we define $C_{c}{ }^{s}$ to be at the occupation probability where the value of $I_{\mathrm{av}}^{\prime}$ is at a maximum. ${ }^{(1)}$ [The variation in the height of the maximum of $I_{\mathrm{av}}^{\prime}$ with different topologies (Fig. 2) is not significant, as it is a simulation artifact due to statistical fluctuations, i.e., it is due to the finite size effects of the sample.]

In Fig. 3 the function $P_{\max }(C)$ [see Eq. (5a)] is shown for four different topologies. Once the value of $C_{c}{ }^{s}$ is known, it is possible to obtain the values of $\bar{P}_{\infty}(C)$ from the $P_{\max }$ curves in Fig. 3. For $C<C_{c}{ }^{s}$ we set $\bar{P}_{\infty}(C)=0$ and for $C>C_{c}{ }^{s}$ we set $\bar{P}_{\infty}(C)=P_{\max }$. Again, Fig. 3 is given mainly for illustration purposes, and as a comparison for the layer and long-range percolation results below, since we give much more refined data in a future publication. 


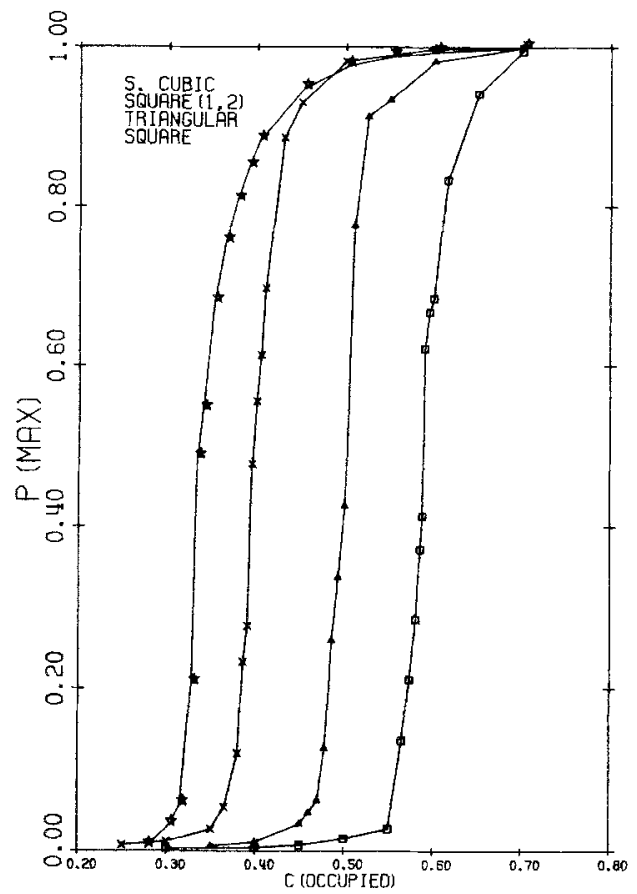

Fig. 3. The probability $P_{\max }$ that an occupied site belongs to the largest cluster [see Eq. (5a)] as a function of the occupied site concentration for four topologies. The square points represent the square lattice, the triangles represent the triangular lattice, the diagonal crosses represent the square $(1,2)$, and the stars represent the simple cubic lattice. The two-dimensional lattices contain $200 \times 200$ sites and the cubic lattice contains $100 \times 100 \times 30$ sites.

\section{SQUARE LATTICE SITE PERCOLATION THRESHOLD}

In order to justify the results discussed below, especially those concerning the critical exponents, we quote here our current results for the critical concentration (site percolation threshold) $C_{c}$ for the square lattice. Table I gives these results. A comparison with literature data is given in Table II.

\section{CRITICAL EXPONENTS FOR THE SOUARE LATTICE AND "CLUSTER MEAN SIZES"}

The critical exponents $\beta, \gamma$, and $\gamma^{\prime}$ have been of much interest in general ${ }^{(16)}$ and in percolation theory in particular. ${ }^{(17,18)}$ We note that one of the simplest tests of scaling theory (or renormalization group theory) ${ }^{(19)}$ is to check the equality

$$
\gamma=\gamma^{\prime}
$$


Table I. Square Lattice Site Percolation Threshold

\begin{tabular}{cccc}
\hline Size & $\begin{array}{c}\text { Number of } \\
\text { configurations }\end{array}$ & $\begin{array}{c}\text { Cyclic boundary } \\
\text { conditions }^{a}\end{array}$ & $C_{c}$ \\
\hline $100 \times 100$ & 80 & No & $0.5905 \pm 0.002^{b}$ \\
$200 \times 200^{a}$ & 10 & No & $0.593 \pm 0.003^{c}$ \\
$400 \times 400$ & 57 & No & $0.5931 \pm 0.0006^{b}$ \\
$1000 \times 1000$ & 30 & No & $0.5931 \pm 0.0006^{b}$ \\
$4000 \times 4000$ & 17 & No & $0.5927 \pm 0.0003^{b}$ \\
\hline
\end{tabular}

"In a few calculations performed with cyclic boundary conditions, the result appeared unchanged within the error limits. In the percolation model, as sites are not correlated, the existence of surfaces does not affect the site distribution. Cluster numbers are marginally affected by the sample surfaces. This effect is roughly proportional to the fraction of the surface sites. In correlated systems such as the Ising model, the role of surfaces is much more pronounced because of the site-site interactions--see Stoll and Schneider. ${ }^{(47)}$

${ }^{b}$ At $68 \%$ confidence.

${ }^{-}$Estimated.

where

$$
I_{\mathrm{av}}(C)=k\left|C-C_{c}^{s}\right|^{-\gamma}, \quad C<C_{c}^{s}
$$

and

$$
I_{\mathrm{av}}^{\prime}(C)=k^{\prime}\left|C-C_{c}^{s}\right|^{-\gamma^{\prime}}, \quad C>C_{c}^{s}
$$

We also define

$$
\bar{P}_{\infty}(C)=k^{\prime \prime}\left|C-C_{c}^{s}\right|^{\beta}
$$

\begin{tabular}{|c|c|c|c|c|c|}
\hline Size & $\begin{array}{l}\text { Number of } \\
\text { configuration }\end{array}$ & $\mathrm{CBC}^{b}$ & $C_{c}$ & Method & Ref. \\
\hline - & - & - & $0.59 \pm 0.1$ & Series & Harris et al. ${ }^{(13)}$ \\
\hline - & - & - & $0.593 \pm 0.002$ & Series & Sykes et al. ${ }^{(14)}$ \\
\hline- & - & - & $0.591 \pm 0.001$ & $\begin{array}{l}\text { Cluster sizes } \\
\text { inter- } \\
\text { polation }\end{array}$ & Stauffer ${ }^{(21)}$ \\
\hline $20 \times 20$ & $100)$ & \multirow{5}{*}{ No } & \multirow{5}{*}{0.595} & \multirow{5}{*}{$\begin{array}{l}\text { Network } \\
\text { simulation }\end{array}$} & \multirow{5}{*}{ Roussenq et al. ${ }^{(15}$} \\
\hline $50 \times 50$ & 100 & & & & \\
\hline $100 \times 100$ & $25\}$ & & & & \\
\hline $300 \times 300$ & $?$ & & & & \\
\hline $1000 \times 1000$ & 5) & & & & \\
\hline
\end{tabular}

Table II. Square Lattice Site Percolation Threshold-(Literature Data) ${ }^{a}$

${ }^{2}$ For further literature data see Refs. 38-44.

${ }^{b}$ Cyclic boundary conditions. 


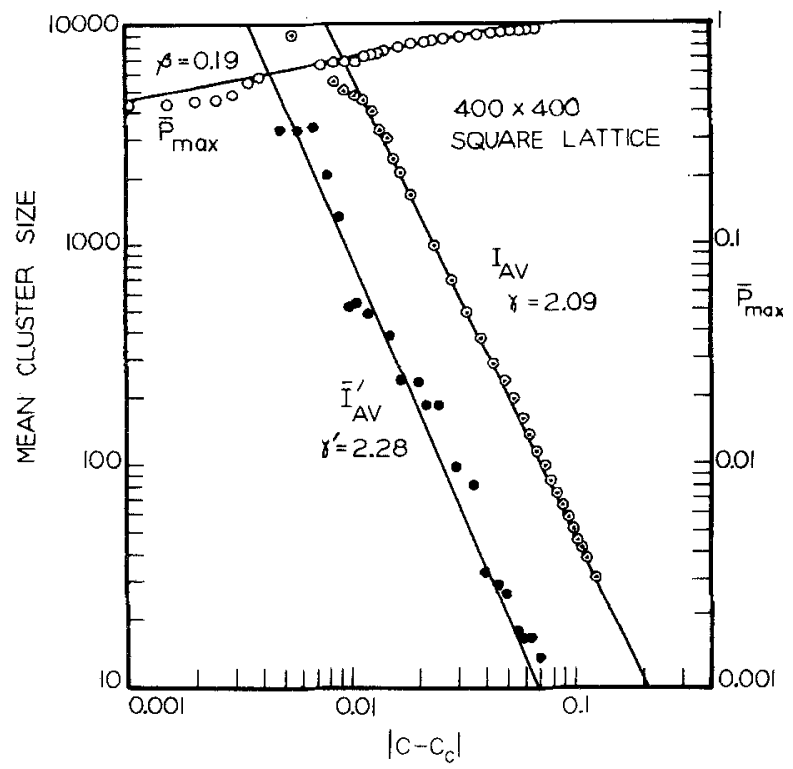

Fig. 4. Critical exponents $\beta, \gamma$, and $\gamma^{\prime}$ for a square lattice of $400 \times 400$ sites. The value of $C_{c}{ }^{s}$ is obtained by first averaging the value $I_{\mathrm{av}}^{\prime}(C)$ for a sample of lattice configurations. The maximum of the $I_{\mathrm{av}}^{\prime}$ distribution is then taken to be the critical site percolation concentration. For the exponents $\beta$ and $\gamma$ the chosen sample of configurations yielded $C_{c}{ }^{s}=0.5935$, while those chosen for the determination of $\gamma^{\prime}$ yielded $C_{c}{ }^{s}=0.5952$. We note that we have used the normalization $\bar{I}_{\mathrm{av}}^{\prime}(C) \equiv\left(1-\bar{P}_{\infty}\right)^{-1} I_{\mathrm{av}}^{\prime}$ for the region $C_{a}>C_{c}^{s}$, where $\gamma^{\prime}$ is the critical exponent. This is very similar to the function defined by Fisher and Essam ${ }^{(34)}$ and by Sykes et al. ${ }^{(35)}$ (However, the latter authors later switched ${ }^{(2 n)}$ to a function equivalent to our ${ }^{(1)} I_{\text {av }}^{\prime}$ )

where

$$
\bar{P}_{\infty}(C) \equiv \begin{cases}P_{\max }(C), & C>C_{c}{ }^{s} \\ 0, & C \gtrless C_{c}{ }^{s}\end{cases}
$$

Logarithmic plots for the relationships of Eqs. (8)-(10) are given in Fig. 4. The results are compared with literature results in Table III.

We conclude from Fig. 4 that, within our uncertainty,

$$
\gamma \simeq \gamma^{\prime}
$$

as expected from scaling theory. More refined values and tests for $\gamma, \gamma^{\prime}, \beta$, and the above equality are in progress.

The ratio of the "cluster mean sizes" above and below $C_{c}$ is given by the ratio of the preexponential factors $k / k^{\prime}$, assuming $\gamma=\gamma^{\prime}$. Our preliminary result for the square lattice is

$$
k / k^{\prime} \approx 16(+83,-9)
$$


Table III. Critical Exponents $\beta, \gamma$, and $\gamma^{\prime}$ for the Square Lattice Site Percolation Problem ${ }^{a}$

\begin{tabular}{lcccl}
\hline \multicolumn{1}{c}{ Method } & $\beta$ & $\gamma$ & $\gamma^{\prime}$ & \multicolumn{1}{c}{ Ref. } \\
\hline Monte Carlo & $0.19 \pm 0.05$ & $2.09 \pm 0.2$ & $2.28 \pm 0.3^{c}$ & Our "preliminary" data \\
Series & $0.148 \pm 0.004$ & $2.3 \pm 0.1$ & - & Harris et al ${ }^{(13)}$ \\
& $0.138 \pm 0.007$ & $2.43 \pm 0.03$ & $1.25-1.75^{c}$ & Sykes et al. ${ }^{(20,25)}$ \\
Monte Carlo & $0.136-0.15$ & $2.3 \pm 0.1$ & & Kirkpatrick $^{(36)}$ \\
\hline
\end{tabular}

${ }^{a}$ For further literature data see Refs. 11, 31, 44-46.

${ }^{b}$ Size: $400 \times 400$.

c Using as definition $\bar{I}_{\mathrm{av}}^{\prime}$ (see text).

${ }^{d}$ Using as definition $I_{\mathrm{av}}^{\prime}$.

which, so far, is practically "consistent" with the two very conflicting literature data of ${ }^{(20)} 2$ and of ${ }^{(21)} 100$. Obviously, the severe statistical scatter is a warning that we should await higher quality data. This is currently underway, using lattices that are two or more orders of magnitude larger. We also notice that while the scaling law $\gamma=\gamma^{\prime}$ is widely accepted, it is not so clear which is the best function for deriving $\gamma^{\prime}$. The different possible definitions (normalizations) for $I_{\mathrm{av}}^{\prime}$ (see caption to Fig. 4) have been pointed out independently by Sykes et al. ${ }^{(20)}$ and by Hoshen et al. ${ }^{(1,26)}$ This problem requires further study.

\section{FRACTIONAL DIMENSION PERCOLATION AND CORRELATION LENGTH EXPONENT}

Do percolation parameters change from two- to three-dimensional lattices "gradually"? An answer to this question can be provided in the context of the "layer cake" problem, i.e., an investigation of "thin crystals" made of $n$ layers, where $n$ ranges from 1 to $L, L$ being the size of the twodimensional lattice forming each layer. This problem has been dealt with previously by Binder, ${ }^{(7)}$ Sur et al., ${ }^{(11)}$ Levinshtein et al., ${ }^{(10)}$ as well as by us. ${ }^{(12)}$ The practical applications of this investigation have ranged from magnets to surface excitons.

Figure 5 illustrates the application of our method, the use of $I_{\mathrm{av}}^{\prime}$ for the determination of the site percolation threshold (critical concentration) $C_{c}$ as a function of the number of square lattice layers. We notice that, except for the top and bottom layers, each lattice site has a simple cubic coordination number of "six." It appears remarkable to us that within about $1 \%$ (the precision of Fig. 5) the $P_{\max }$ curve for such a 20-layer "cake" $(n=20)$ is the same as that for a simple cubic lattice cube $(n=100)$. Obviously, the $I_{\mathrm{av}}^{\prime}$ 


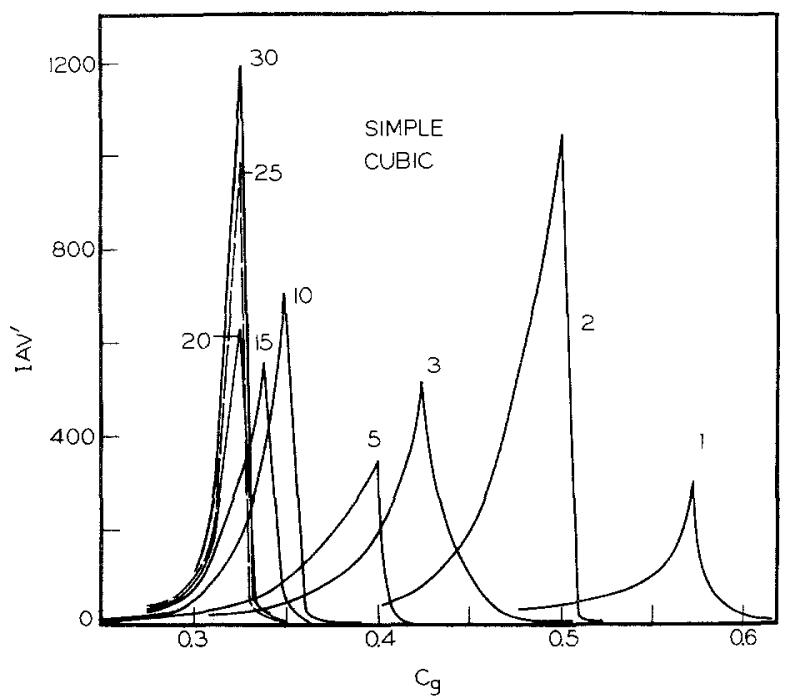

Fig. 5. The reduced average cluster size $I_{\mathrm{av}}^{\prime}$ vs. the occupied site (guest) concentration $C_{g}$ for a three-dimensional simple cubic lattice of $100 \times 100 \times n$ sites. The maxima in the plots give the percolation concentrations $\left(C_{c}{ }^{s}\right)$ for various values of the third dimension parameter $n$.

peaks in Fig. 5 become sharper with increasing $n$, simply because of the concomitant increase in the total number of sites $n$.

The expected ${ }^{(7-11)}$ power law for the effect of $n$ on $C_{c}$ is given by

$$
n=A\left|C_{c}{ }^{n}-C_{c}{ }^{\infty}\right|^{-v}
$$

where $\nu$ should be the correlation length exponent. Figure 6 is based on our data for $400 \times 400$ layers $(L=400)$ with no periodic boundary conditions. We get the values $\nu=0.92$ and $A=0.38$ (correlation 0.990 ). If we exclude the $n=64$ point (due to its lower statistical quality and closeness to the "not quite infinite" value of $n=400$ ), we get $\nu=0.98, A=0.33$ (correlation 0.995). Weighting even heavier the smaller lattices gave $\nu=1.04$ (Fig. 6). Comparisons to previous work are given in Table IV.

Table IV. Critical Exponent $\nu$ (Three Dimensions)

\begin{tabular}{lll}
\hline \multicolumn{1}{c}{ Method } & \multicolumn{1}{c}{ Value } & \multicolumn{1}{c}{ Ref. } \\
\hline Monte Carlo & $0.9 \pm 0.05$ & Levinshtein et al. ${ }^{(10)}$ \\
Conductivity (and scaling) & 1 & Shur (22) \\
Series expansion & $0.82 \pm 0.05$ & Dunn et al. (23) $^{\text {Monte Carlo (finite size scaling) }}$ \\
Monte Carlo ("layer cake") & $0.8 \pm 0.1$ & Sur et al..$^{(1)}$ \\
\hline
\end{tabular}




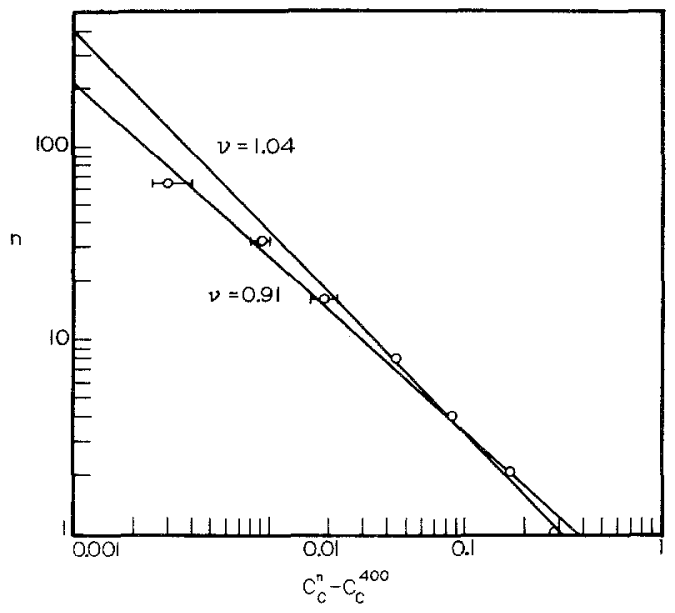

Fig. 6. Critical exponent $\nu$ from the layer cake percolation. This is based on the assumption that $C_{c}{ }^{400}$ is close to $C_{c}{ }^{\infty}$. However, while for $n \rightarrow \infty$ one has $\left|C_{c}{ }^{n}-C_{c}{ }^{\infty}\right| \rightarrow 0$, this is not correct for $\left|C_{c}{ }^{n}-C_{c}^{400}\right|$. Therefore we did put maximum weight on points where $n \ll 400$, in addition to the obviously lower uncertainty for these points due to (a) the larger absolute value of $\left|C_{c}{ }^{n}-C_{c}^{400}\right|$ and (b) the larger number of configurations used in our work for these points, due to reduced computational costs. Note also that when uncertainty limits are absent, they are about equal to or less than the size of the circle. They are significantly lower for $n=1,2$.

To further test the consistency of our results $\left(C_{c}{ }^{n}, v\right)$, we plot a "finite size scaling curve" in analogy to Sur et al. ${ }^{11}$ We emphasize here the following:

1. The finite "size" of the sample (Sur et al.'s $L$ ) is the number of layers $n$ (each layer being of size $400 \times 400$ ).

Fig. 7. Test for finite-size scaling for $P_{n}(C)$. Here $C_{c}=0.3135, \beta=0.35$, and $\nu=0.97$ (see text). The codes for $n=32$, 64,128 , and 400 are given in the figure. Note that here we "scale" only one dimension (the layer number $n$ ), in contrast to all three dimensions $(L)$ in the similar plot of Sur et al. ${ }^{(11)}$ Only one $400^{3}$ configuration was used. We thank one referee for suggesting this plot.

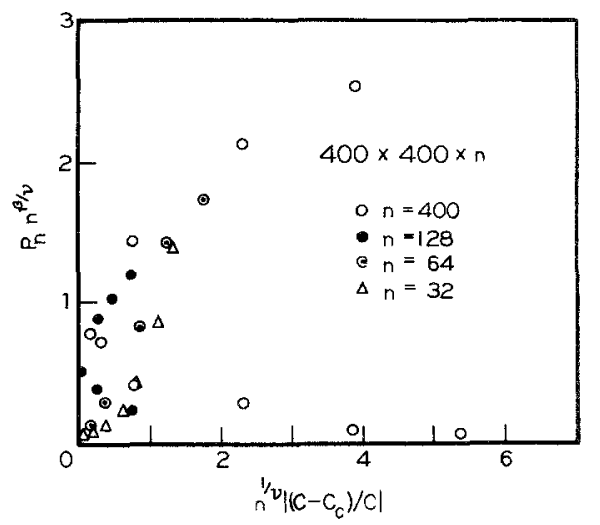


2. We have determined our critical concentration $C_{c}{ }^{n}$ a priori for each layer parameter $n$ via our method of finding the $I_{\mathrm{av}}^{\prime}$ peak.

3. We have not used subjective techniques to minimize the scatter of the points from an "imaginary" scaling function $X\left(X_{1}\right.$ of Sur et al.). (11)

4. We have used the value of $\beta=0.35$ taken from Shur. ${ }^{(22)}$

5. We have used our values of $C_{c}=0.3135$ and $\nu=0.97$.

This scaling plot is given in Fig. 7. We note that, using the values $\beta=0.41$ and $C_{c}=0.3115$ from Sur et al. ${ }^{(11)}$ we got a significantly higher scatter of points ("as discerned by the eye"). We are not quite sure what the significance

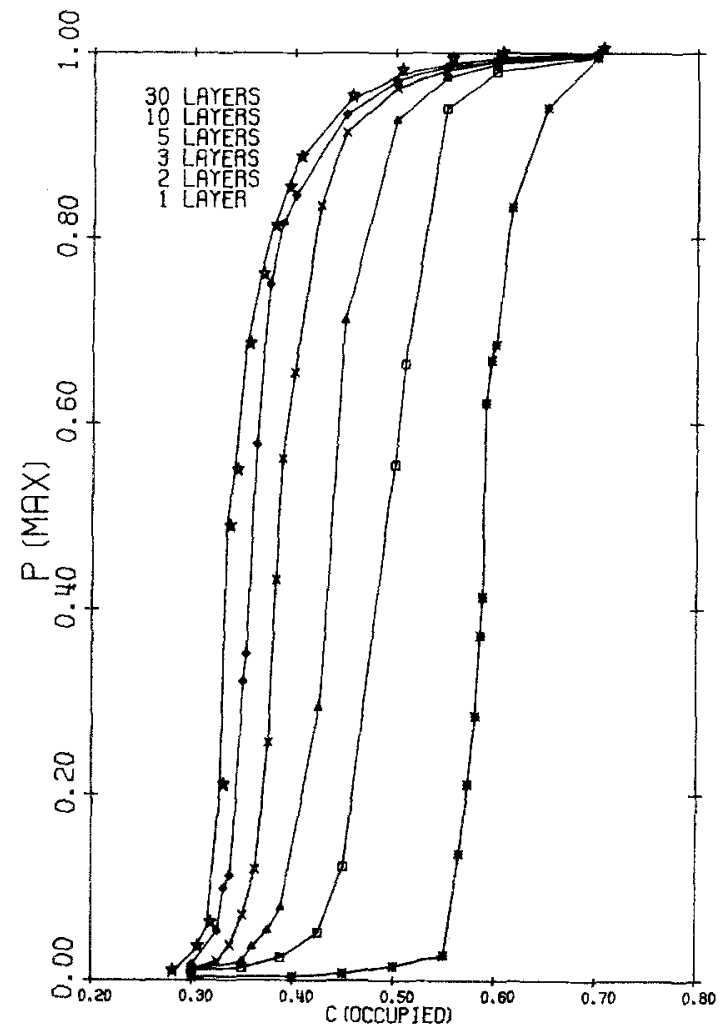

Fig. 8. The probability $P_{\max }$ that an occupied site belongs to the largest cluster, as a function of the occupied site concentration for a three-dimensional simple cubic lattice containing $100 \times 100 \times n$ sites. The layer index $n$ is varied from 1 (simple twodimensional square lattice), as represented by the asterisks, to 30 , represented by the five-pointed stars. (Larger $n$ values give identical curves, within the precision of this figure.) Note that as the thickness of the lattice increases, the $P_{\max }$ curves shift from right to left on the concentration axis. 
of the above consistency test is and to what extent it really discriminates between the results of Shur, ${ }^{(22)}$ Sur et al., ${ }^{(11)}$ and ours.

For future reference and potential use by experimentalists we give here some rough curves of the maxicluster probability $P_{\max }(C)$ with the number of layers $n$ as a parameter (Fig. 8). We also quote here the value of $C_{c}{ }^{\infty}=$ $0.310 \pm 0.004$, derived from series expansions of the mean cluster size. ${ }^{(24)}$

\section{LONG-RANGE INTERACTIONS AND CONTINUOUS PERCOLATION}

In order to look at percolation due to long-range interactions, some slight modifications of the previous notations are necessary. We can define an integer quantity $S_{\max }$ as the maximum number of successive lattice constants over which an interaction between two sites can occur. In a square lattice, the values of $S_{\max }$ map out diamond-shaped regions of interaction, denoting the number of neighbors of a given site. Figure 9 shows graphically what this region of interaction looks like. The number of sites in this interaction zone is given by

$$
M=\left(S_{\max }+1\right)^{2}+S_{\max }^{2}
$$

The number of neighbors for a given site is then $M-1$. Thus, the trivial case of the square lattice with nearest neighbor interactions only, with $S_{\max }=1$, has four interacting sites. The CMLT is easily adapted to search for these longer range interactions and thus $I_{\mathrm{av}}^{\prime}$ values can be calculated for various guest concentrations, yielding values of $C_{c}{ }^{s}$ for each given $S_{\max }$. The results

Fig. 9. A representative portion of a two-dimensional square lattice, where the maximum number of successive nearest neighbor bonds over which an interaction between two sites can occur, $S_{\max }$, is equal to six. The number of sites included in the interaction region is equal to $M=S_{\max }^{2}+\left(S_{\max }+1\right)^{2}$.

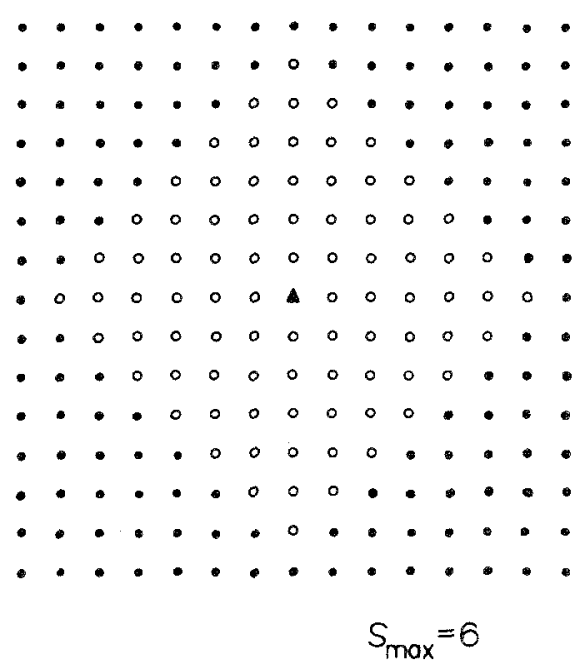




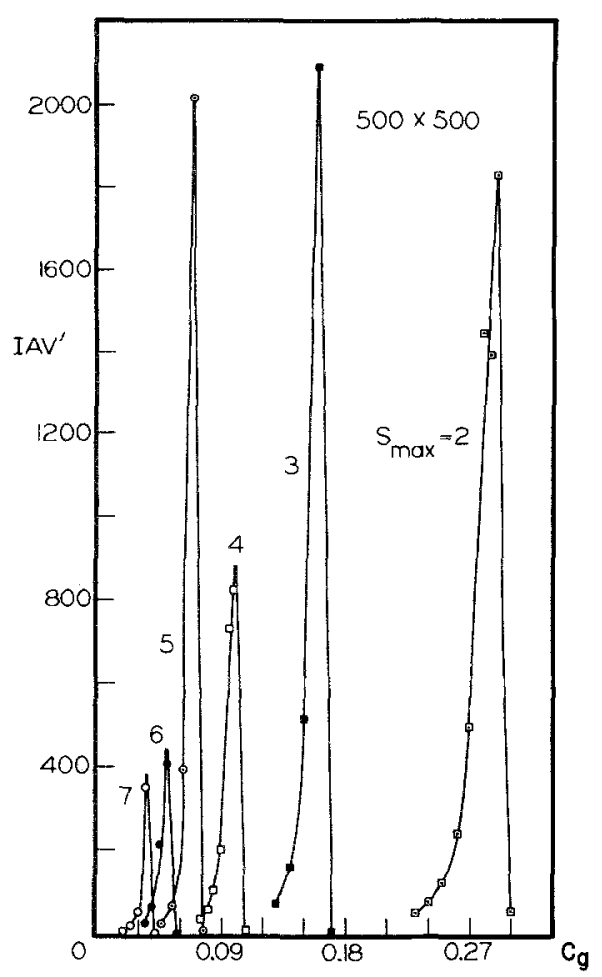

Fig. 10. Long-range percolation for a square lattice. $S_{\max }$ is the maximum number of successive nearest neighbor bonds over which an interaction between two sites can occur (see Fig. 9). $I_{a v}^{\prime}$ is the reduced average cluster size. $C_{g}$ is the fraction of occupied sites (guests). The discontinuity in the $I_{\mathrm{av}}^{\prime}$ vs. the molar guest concentration $C_{g}$ curve gives the critical percolation concentration, for a lattice of $500 \times 500$ sites. The differences in the ordinate value of $l_{\mathrm{av}}^{\prime}$ for different $S_{\max }$ are not significant, due to statistical fluctuations. Results for $S_{\max }>7$ are not shown here.

of these simulations are shown in Figs. 10 and 11 for values of $S_{\max }$ up to 7 and 8 , respectively. We have calculated $C_{c}{ }^{s}$ for values of $S_{\max }$ up to 12 , where 313 sites are included in the zone of interaction. In order to compare our results for long-range interactions with the results given by Pike and Seager ${ }^{(5)}$ for the continuous percolation problem, we shall assume that the simulated lattice, which contains $N$ sites, occupies a unit area. Thus, the site density for the concentration $C$ is $G=N C$. Now we shall define a radius of interaction $\rho$ given by

$$
\rho=(M / \pi N)^{1 / 2}
$$

where $\rho$ is given in terms of the number of neighbor sites $(M-1)$ as defined in Eq. (14). Following Pike and Seager, we shall define a parameter $r_{s}$ corresponding to an average distance between occupied sites:

$$
r_{s}=(\pi N C)^{-1 / 2}
$$

Now $\rho$ can be rewritten as a dimensionless quantity $R$,

$$
R=\rho / 2 r_{s}=(M C)^{1 / 2} / 2
$$


Fig. 11. The critical percolation concentration $C_{c}{ }^{s}$ vs. the range of extended interaction $S_{\max }$ (see Fig. 10). The size of the lattice was $500 \times 500$ sites. (For $S_{\max }=9$ and 10, see Fig. 12.)

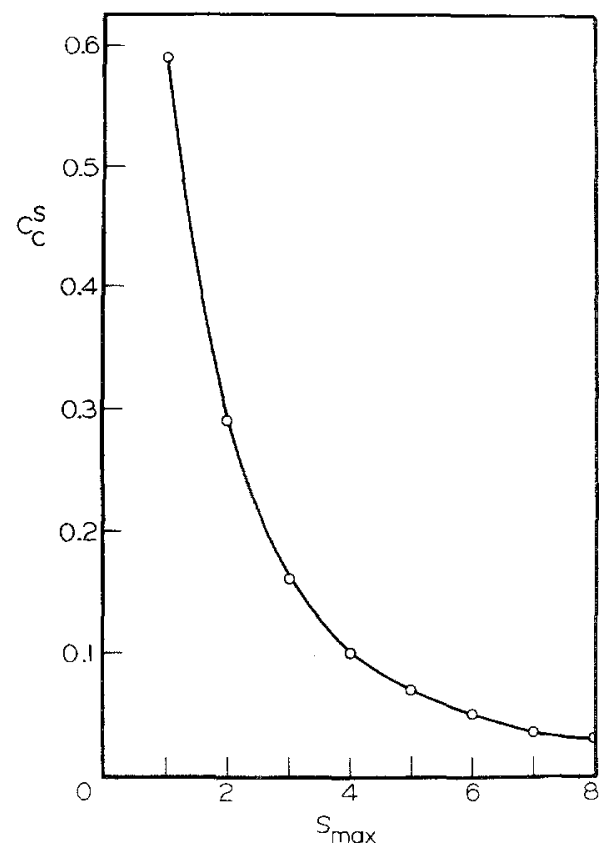

and a critical radius $R_{c}$ can be defined in terms of $C_{c}^{s}$. Hence, the following relationship is obtained from Eq. (17):

$$
M^{1 / 2}=2 R_{c}\left(C_{c}^{s}\right)^{-1 / 2}
$$

In Fig. 12, $M^{1 / 2}$ is plotted vs. $\left(4 / C_{c}^{s}\right)^{1 / 2}$. This curve exhibits remarkable linearity for $S_{\max }>2$, confirming previous estimates ${ }^{(5)}$ on the value of $R_{c}$, which we find to be $\sim 1.0$. The lack of dependence of the continuous percolation parameter $R_{c}$ on the interaction distance is a strong indication that the particular lattice structure has virtually no effect on these longer range values of $C_{c}{ }^{s}$. We thus get, for two-dimensional square lattices,

$$
\begin{aligned}
C_{c}^{s} & =4 R_{c}{ }^{2} M^{-1} \\
C_{c} s\left(S_{\max }\right) \approx 4 M^{-1} & =4 /\left[S_{\max }^{2}+\left(S_{\max }+1\right)^{2}\right] \text { iff } S_{\max }>2
\end{aligned}
$$

A similar derivation is easily carried through for the three-dimensional case. The result is

$$
M^{1 / 3}=2 R_{c}\left(C_{c}^{s}\right)^{-1 / 3}
$$

Here one can plot $M^{1 / 3}$ vs. $\left(8 / C_{c}\right)^{1 / 3}$ and find the $R_{c}$ parameter. According to Pike and Seager, ${ }^{(5)}$ we expect $R_{c} \approx 0.7$ for the three-dimensional case. (We 


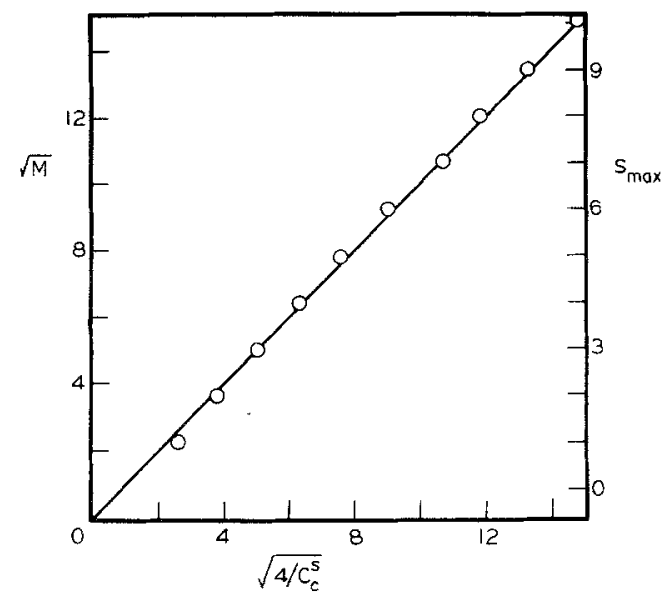

Fig. 12. A plot of $M^{1 / 2}$ [given by Eq. (14)] vs. $\left(4 / C_{\mathrm{c}}\right)^{1 / 2}$ [see Eq. (18)].

plan to check this out.) We also note that for a simple cubic lattice we get (analogously to Eq. (14)):

$$
M=\frac{1}{3}\left(2 S_{\max }+1\right)\left(2 S_{\max }^{2}+2 S_{\max }+3\right)
$$

This gives, for a simple cubic lattice,

$$
C_{c}^{s}\left(S_{\max }\right)=8 R_{c}^{3} M^{-1}=24 R_{c}^{3} /\left[\left(2 S_{\max }+1\right)\left(2 S_{\max }^{2}+2 S_{\max }+3\right)\right]
$$

\section{LONG-RANGE PERCOLATION AND CRITICAL EXPONENTS}

It is usually assumed ${ }^{(16)}$ that classical "mean field" theories are valid provided that the interaction range is large. We have thus been encouraged by one referee to list here our results for large $S_{\max }$. We give some preliminary results in Fig. 13. The important results are:

1. The exponent $y$ is the same, within the statistical uncertainties, for all $S_{\max }$ values $2-7$. The average result $(2.1 \pm 0.3)$ is practically the same as our result given above for $S_{\max }=1$, i.e., the simple square lattice case (coordination number 4), where $\gamma=2.1$.

2. The same is true for $\beta$, even though here, for lack of enough data points, we had to scramble the results for all values of $2 \leqslant S_{\max } \leqslant 7$. This should not be bad, assuming that the individual slopes are parallel. Consulting the data points of Fig. 13, our argument seems convincing. Here we note that 


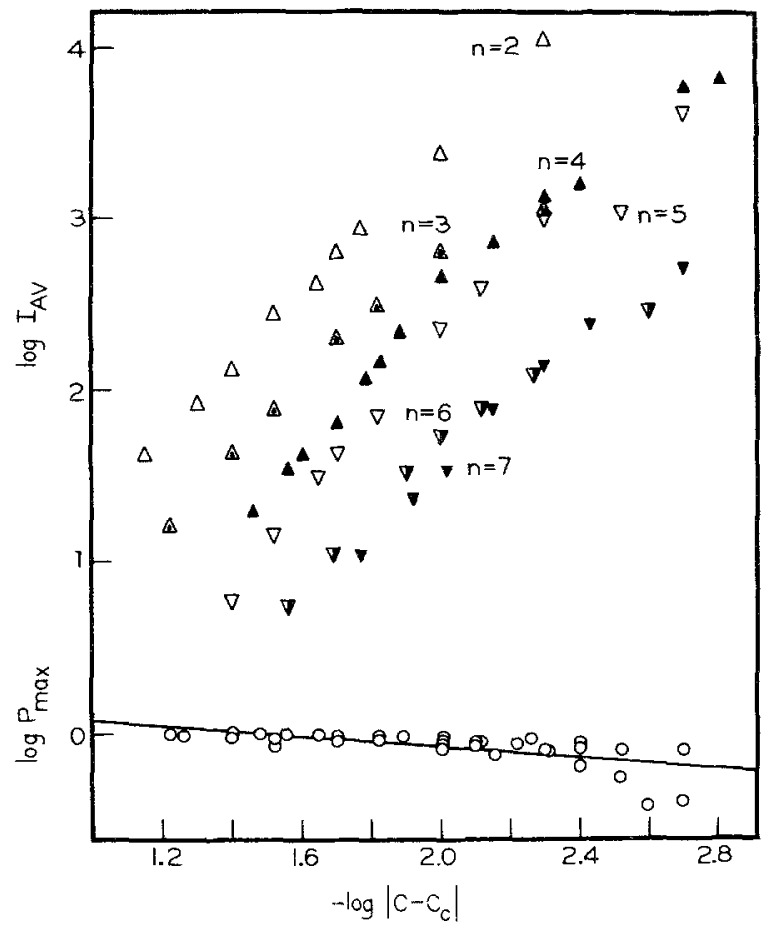

Fig. 13. Critical exponents $\gamma$ and $\beta$ for long-range percolation in two dimensions. The parameter $n$ is actually $S_{\max }$ (see text), not to be confused with the number of layers $n$ used before. The overall values $\gamma=2.1$ and $\beta=0.14$ are preliminary (see text). The lattice size is $500 \times 500$ (and the typical number of configurations is three). Here $\log =\log _{1.0}$.

the slope $\beta=0.14$ is actually in excellent agreement with the values for short range two-dimensional lattices (Table III).

3. We have a very preliminary value for $\gamma^{\prime}$, also derived by "scrambling" the results for $2 \leqslant S_{\max } \leqslant 7$. While the scatter is large, we give a tentative value of $2.15 \pm 0.5$.

4. The above results, assuming scaling, ${ }^{(17)}$ give other exponents, like $\delta=1+\gamma / \beta=16$. $^{(25)}$

5. Our results for $\gamma, \gamma^{\prime}$, and $\beta$ are extremely far from the classical ${ }^{(16,17)}$ limiting values of unity. On the other hand, they are practically indistinguishable from the simple square lattice results. It is not clear to us whether the observation of a crossover to mean field exponents requires just an order of magnitude (or two) larger interaction distance ${ }^{(27)}$ or also a nonabrupt interaction cutoff. ${ }^{(28)}$ 


\section{TERNARY LATTICES AND EXCITON PERCOLATION}

The problem of exciton supertransfer in a binary lattice that also contains a small fraction of exciton sensor has been described by us previously. ${ }^{(12)}$ The formalism has been worked out specifically for Frenkel excitons, i.e., electronic, vibrational, or vibronic excitations of molecular "parentage" in molecular solids. The exciton is usually assumed to be localized at one "guest" site at a time and to move (coherently, stochastically, or "inbetweenly") from one guest site to the next. However, the exciton is excluded from visiting "host" sites due to energy considerations. Thus, the "guest" site can be considered as an exciton carrier and the "host" site as an exciton barrier. However, in addition, a small fraction of the guest sites are distinct and designated as sensors. When the exciton visits such a sensor ("supertrap") site it may be captured ("trapped") irreversibly. This act is also called "registration," as the captured exciton decays with a well-known probability and this decay is monitored experimentally (i.e., via the radiative decay). Usually the "host," the ordinary "guest," and the "sensor" guest sites are occupied by molecules that differ from each other only by isotopic or minor chemical substitution, and thus the lattice topology is assumed to be unchanged from that of the pure "guest" (or "host") crystal under the same thermodynamic conditions. The supertransfer limit is that limit where the exciton lifetime is long enough to permit registration provided only that there exists a topological "guest bridge" connecting the original site of the exciton with at least one sensor site. Alternatively, the "bridge topology" may be defined so as to guarantee sufficient registration time.

The probability $P$ of exciton transfer and registration at the sensor is ${ }^{(12)}$

$$
\begin{gathered}
P=\sum_{m}\left[1-(1-m / G)^{z}\right] i_{m} m / G=1-\sum_{m}(1-m / G)^{\mathrm{z}} i_{m} m / G \\
\text { iff } 1 \ll Z \ll G
\end{gathered}
$$

where $m$ is the size of a cluster, $i_{m}$ is the frequency of the cluster size $m, G$ is the number of guests, and $Z$ is the number of sensors (assuming a trapping efficiency of unity).

In the thermodynamic limit of large ("infinite") samples, the finite clusters have a limited size $m$, whereas the numbers $G$ and $Z$ (and the cluster number $i_{m}$ ) approach infinity. Then

$$
\lambda^{m} \equiv(1-m / G)^{Z}=e^{-m Z / G}=e^{-m C_{s} / C_{g}}
$$

or, for the finite clusters,

$$
\lambda=e^{-Z / G}=e^{-C_{s} / C_{g}} \leqslant 1
$$

and $C_{s}$ and $C_{s}$ are, respectively, the guest (total guest) and sensor concentra- 
tions (mole fractions). Noting that $\lambda^{m} \rightarrow 0$ for the infinite cluster, we can infer that $\lambda$ is given by Eq. (25) in the following expression derived from Eq. (23):

$$
P=P(C, \lambda)=1-G^{-1} \sum_{m} i_{m} m \lambda^{m}
$$

In this way the parameter $\lambda$, introduced mathematically by Gaunt and Sykes, ${ }^{(25)}$ is identified with an experimental quantity. ${ }^{2}$ We also note that the ratio $Z / G=C_{s} / C_{g}$ corresponds to a "notional" or effective field, i.e., the magnetic field in other cluster models ${ }^{(10)}$ (we are indebted to one referee for the above points).

The function $P=P\left(C_{g}, \lambda\right)$ has been shown ${ }^{(25)}$ to exhibit a dominant critical point singularity at the critical concentration $C_{c}$ :

$$
P_{c}(\lambda) \equiv P\left(C_{c}, \lambda\right) \propto E(1-\lambda)^{1 / \delta}
$$

where the amplitude $E$ has been estimated ${ }^{(25)}$ to be about 1.1 and $\delta$ is about 16 (see above) or $^{(26)} 18$. Figures 14 and 15 demonstrate the behavior of $P\left(C_{g}, \lambda\right)$ for various values of the parameter $C_{s}$, which in turn specifies $\lambda$. Using Eq. (26a), with $C_{c}=0.593$ and $\delta=18$, we calculate $P_{c}(\lambda)$ to be $0.66,0.71,0.73,0.78$, and 0.82 for the $C_{s}$ values of $0.0002,0.0006,0.001,0.004$, and 0.008 , respectively. These calculated values are consistent with the values of $P_{c}$ in Figs. 14 and 15, considering the large uncertainties involved (both in the Ref. 25 parameters and in these crude simulations).

Using the CMLT, we can obtain the values of $m$ and $i_{m}$ for a given binary lattice and then calculate the value of $P$ as a function of $C_{g}$ for different sensor concentrations. If we consider only the regions where $C_{g} \gg C_{c}{ }^{s}$ or $C_{g} \ll C_{c}^{s}$, where there are not many large clusters, then we can simplify Eq. (23) to ${ }^{(12 a)}$ [compare also Eqs. (24), (25)]

$$
P=\bar{P}_{\infty}+\left(Z / G^{2}\right) \sum_{m \neq m^{\prime}} i_{m} m^{2}
$$

Using the definition of the average cluster size [Eq. (6)], we arrive at the simple expression $^{(12 c)}$

$$
P=\bar{P}_{\infty}+Z G^{-1} I_{\mathrm{av}}^{\prime}
$$

These percolation probabilities are given in Fig. 14 for four different sensor concentrations. The effect of the sensor concentration on the percolation probability is the largest for guest concentrations just below percolation but is negligible above the critical percolation concentration, in the domain of supertransfer. ${ }^{(12)}$ As the sensor concentration is decreased, the probability of an exciton registering at a scarce sensor site approaches that of $\bar{P}_{\infty}$, as only in an "infinite" cluster is there an appreciable probability for a cluster to contain a sensor. Equation (23) is a good approximation only when $Z \ll G$.

2 Note that, below percolation, $\partial P\left(C_{g}, S\right) /\left.\partial S\right|_{S \rightarrow 0}=I_{\text {av }}\left(C_{g}\right)$, where $S=Z / G$, while above percolation this derivative is equal to $I_{\mathrm{a} v}^{\prime}(C)$. 


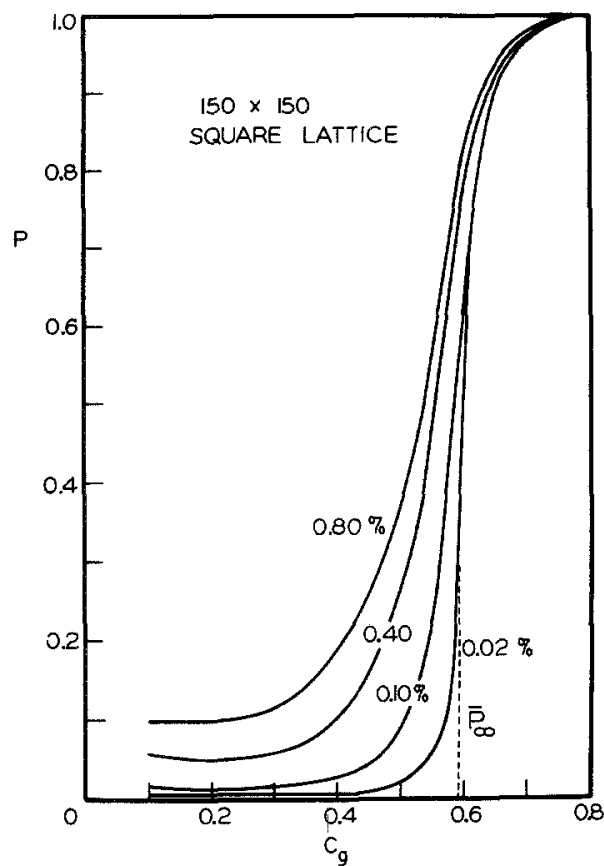

Fig. 14. Exciton supertransfer probability $P$ vs. guest concentration $C_{g}$ (mole fraction) with sensor concentration $C_{s}$ as parameter (all concentrations in mole percent). These $150 \times 150$ square lattice curves are calculated from Eq. (23). The $\bar{P}_{\infty}$ curve is calculated from Eq. (5a). Note that a random number generation subroutine was used to form a random binary system, independent of the value of $C_{s}(=Z / N)$.

Thus, for the high sensor concentration, we get abnormally high values of $P$ for $C_{g} \ll C_{c} s$ in Fig. 14.

An alternative and more empirical method of approaching this problem is illustrated in Fig. 15. In addition to the binary lattice used in the CMLT, we label $Z=C_{s} N$ sites as sensors and then calculate $P_{y}$, the fraction of the total guest that is connected via a succession of nearest neighbor guest interactions to a sensor. With the assumption of supertransfer, we would expect that, for low sensor concentrations, the two curves $P_{y}$ and $P$ should be equivalent. The function $P_{y}$ does not run into problems at high $C_{s}$, unlike the function $P$, because there are no approximations involved in its evaluation. ${ }^{3}$

${ }^{3}$ We note that we give here a simple topological interpretation to the generating function $^{(25)} \lambda$ and the probability ${ }^{(25)} P(C, \lambda)$. The latter is the probability that any cluster contains a "sensor," where this sensor concentration (in the "guest") is $S=-\log \lambda$. We have recently used an even simpler approach, where $\lambda \equiv 1-S$ is simply the "non-sensor" site concentration. This gives similar results, but $E \rightarrow 1$ as $\lambda \rightarrow 0$ (i.e., $S \rightarrow 1$ ). 


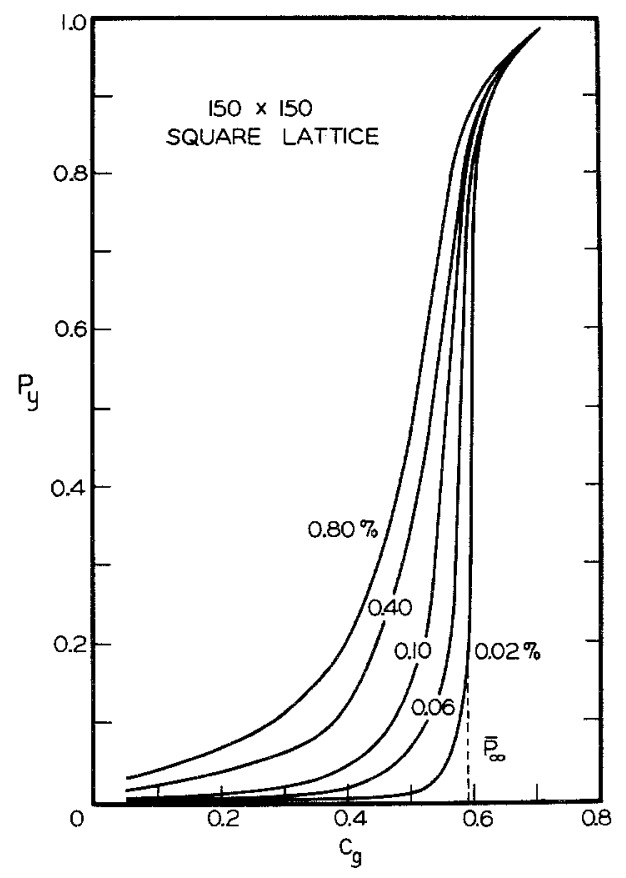

Fig. 15. A computer simulation of the exciton supertransfer probability $P_{y}$ vs. guest concentration $C_{g}$ (mole fraction) with sensor concentration $C_{s}$ (mole percent) as a parameter. The $150 \times 150$ square lattice is generated by the computer, with the help of a random number generating subroutine, as a ternary system, including $C_{s}$ mole percent of a supertrap (sensor). $P_{y}$ is the fraction of total guest that is connected via a succession of nearest neighbor guest interactions to a sensor.

Both above approaches are valid only in the exciton supertransfer limit, ${ }^{(12)}$ i.e., where no dynamic (time) constraints are involved. This is often a reasonable assumption for real systems. ${ }^{(12)}$ It is also useful as a check on timedependent calculations, ${ }^{(12)}$ providing a convenient limiting behavior. [On the other hand, this limit is correct "by definition" if the cluster connectivity is defined by the available time (see above).]

We briefly illustrate here an application of Eq. (18) to the Frenkel exciton superexchange problem. ${ }^{(3)}$ First, we solve the quadratic equation (19b) and get, for the square lattice case,

$$
\bar{n} \equiv S_{\max } \approx\left[-1+\left(-1+8 / C_{c}\right)^{1 / 2}\right] / 2
$$

Now, the exciton superexchange interaction in a typical isotopically doped crystal is $^{(12)}$

$$
J_{0, n}=\Gamma_{n} \beta^{n} / \Delta^{n-1}
$$


where $\Gamma_{n}$ is the number of paths, involving $n$ lattice constants, between guest site 0 and a guest site being $n$ lattice constants away. $\beta$ is the exciton exchange interaction (for one lattice constant) and $\Delta$ is the "trap depth" (guest-host energy separation in the ideal mixed crystal). ${ }^{(29)}$ In an experimental situation where time (or other considerations) ${ }^{(3)}$ determines the smallest effective value of $J\left(=\bar{J}_{0, \bar{n}}\right)$, it does determine a cutoff value for $n$, designated as $\bar{n}$. One can thus easily connect $\bar{J}$ to a critical percolation concentration $C_{c}$, by combining Eqs. (29) and (30). This relationship is important for anisotropic crystals, where the exciton exchange is essentially two-dimensional. ${ }^{(3,29)}$ The extension to isotropic (i.e., simple cubic) cases is straightforward.

\section{DISCUSSION}

In recent years more information has become available on the critical behavior of percolating systems. Although exact methods are not available for the determination of the critical percolation parameters for real lattices, some dimensional invariants have been determined. These include the above discussed critical radius ${ }^{(5)}$ and the critical volume ${ }^{(30)}$ for continuous systems, as well as the exponential scaling factors discussed above.

An interesting problem is to determine the change of the universal quantities $^{(31)}$ upon changing the dimensionality of lattices, i.e., by observing the change in the universal quantities as the number of two-dimensional layers increases. It is also possible to proceed continuously from one lattice topology to another by assigning bond probabilities to some nearest neighbor bonds. Thus, if the two bonds perpendicular to a crystal plane in a simple cubic lattice were assigned a bond probability $p$, where $0 \leqslant p \leqslant 1$, we would have a continuous transition from a cubic three-dimensional lattice, where $p=1$, to a planar square lattice, where $p=0$. In this case we would be dealing with a generalized site-bond percolation problem. In a future paper ${ }^{(33)}$ (III), we shall be discussing algorithms on percolation and cluster size distribution for the generalized site-bond problem. Finally, we would like to emphasize the limited relationship between our ternary system functions and the recently discussed "polychromatic" percolation. ${ }^{(37)}$

\section{ACKNOWLEDGMENTS}

We would like to thank Paul Klymko for his help with the calculations involving the critical exponents and critical concentrations for both the twodimensional and the layer problems. We also like to thank Jim Newhouse for his help with the data handling of the long-range problem. We are also very much indebted to one of the referees for making numerous and valuable suggestions, bringing to our attention many references, and especially for 
pointing out to us the analogy between the exciton percolation model and the cluster model involving magnetic fields, i.e., Eq. (24).

\section{REFERENCES}

1. J. Hoshen and R. Kopelman, Phys. Rev. B 14:3438 (1976).

2. J. M. Hammersley and D. C. Handscomb, in Monte Carlo Methods (Methuen, London, 1964), chap 11; V. S. Shante and S. Kirkpatrick, Adv. Phys. 20:325 (1971); J. W. Essam, in Phase Transitions and Critical Phenomena, C. Domb and M. S. Green, eds. (Academic, New York, 1973); S. Kirkpatrick, Rev. Mod. Phys. 45:574 (1973).

3. R. Kopelman, E. M. Monberg, F. W. Ochs, and P. N. Prasad, J. Chem. Phys. 62:292 (1975); R. Kopelman, E. M. Monberg, and F. W. Ochs, Chem. Phys. 19:413 (1977).

4. A. S. Skal, B. I. Shklovski, and A. L. Efros, Sov. Phys.-Solid State 15:961 (1973); Fiz. Tverd. Tela 15:1423 (1973).

5. G. E. Pike and C. H. Seager, Phys. Rev. B 10:1421, 1435 (1974).

6. R. Zallen and H. Scher, Phys. Rev. B 4:4471 (1971).

7. K. Binder, Thin Solid Films 20:367 (1974).

8. M. E. Fisher, in Proc. Intern. School of Physics "Enrico Fermi" on Critical Phenomena, Course 51, M. S. Green, ed. (Academic Press, New York, 1971).

9. M. E. Fisher and M. N. Barber, Phys. Rev. Lett. 28:1516 (1972).

10. M. E. Levinshtein, B. I. Shklovskii, M. S. Shur, and A. L. Efros, Sov. Phys.-JETP 42:197 (1976); Zh. Eksp. Teor. Fiz. 69:386 (1975).

11. A. Sur, J. L. Lebovitz, J. Morr, M. H. Kalos, and S. Kirkpatrick, J. Stat. Phys. 15:345 (1976).

12. (a) J. Hoshen and R. Kopelman, J. Chem. Phys. 65:2817 (1976); (b) R. Kopelman, E. M. Monberg, and F. W. Ochs, Chem. Phys. $21: 373$ (1977); (c) R. Kopelman, J. Phys. Chem. 80:2191 (1976); (d) R. Kopelman, in Radiationless Processes in Molecules and Condensed Phases (Vol. 15 of Topics in Applied Physics), F. K. Fong, ed. (Springer, Berlin, 1976); (e) R. Kopelman, 8th Molecular Crystal Symp., Santa Barbara, California (1977).

13. A. B. Harris, T. C. Lubensky, W. K. Holcomb, and C. Dasgupta, Phys. Rev. Lett. 35:327 (1975).

14. M. F. Sykes, D. S. Gaunt, and M. Glen, J. Phys, A 9:97 (1976).

15. J. Roussenq, J. Clerc, G. Giraud, E. Guyon, and H. Ottavi, J. Phys. (Paris) 37:L99 (1976).

16. L. P. Kadanoff, W. Gotze, D. Hamblen, R. Hecht, E. A. S. Lewis, V. V. Palciauskas, M. Rayl, J. Swift, D. Aspnes, and J. Kane, Rev. Mod. Phys. 39:395 (1967).

17. J. W. Essam and K. M. Gwilym, J. Phys. C 4:L228 (1971).

18. A. G. Dunn, J. W. Essam, and J. M. Loveluck, J. Phys. C 8:743 (1975).

19. M. E. Fisher, Rev. Mod. Phys. 46:597 (1974).

20. M. F. Sykes, D. S. Gaunt, and M. Glen, J. Phys. A 9:725 (1976).

21. D. Stauffer, $Z$. Physik B 25:391 (1976).

22. M. S. Shur, J. Phys. C 9:L229 (1976).

23. A. G. Dunn, J. W. Essam, and D. S. Ritchie, J. Phys. C 8:4219 (1975).

24. M. F. Sykes, D. S. Gaunt, and M. Glen, J. Phys. A 9:1705 (1976).

25. D. S. Gaunt and M. F. Sykes, J. Phys. A 9:1109 (1976).

26. J. Hoshen, P. Klymko, and R. Kopelman, unpublished.

27. D. S. Gaunt, M. F. Sykes, and H. Ruskin, J. Phys. A 9:1899 (1976).

28. R. Zallen, private communication, October 1977. 
29. R. Kopelman, Excitons in Pure and Mixed Molecular Crystals, in Excited States II, E. C. Lim, ed. (Academic Press, New York, 1975).

30. H. Scher and R. Zallen, J. Chem. Phys. 53:3759 (1970).

31. D. Stauffer, Phys. Rev. Lett. 35:394 (1975).

32. M. F. Sykes, D. S. Gaunt, and M. Glen, J. Phys. A 9: L43 (1976).

33. J. Hoshen, unpublished.

34. M. E. Fisher and J. W. Essam, J. Math. Phys. 2:609 (1961).

35. M. F. Sykes, D. S. Gaunt, and M. Glen, J. Phys. A 9:715 (1976).

36. S. Kirkpatrick, Phys. Rev. Lett. 36:69 (1976).

37. R. Zallen, Phys. Rev. B 16:1426 (1977).

38. H. L. Frisch, E. Sonnenblick, V. A. Vyssotsky, and J. M. Hammersley, Phys. Rev. 124:1021 (1961).

39. P. Dean, Proc. Camb. Phil. Soc. 59:397 (1963).

40. P. Dean and N. F. Bird, National Physical Laboratory, Report No. Ma61, unpublished (1966); Proc. Camb. Phil. Soc. 63:477 (1967).

41. M. F. Sykes and J. W. Essam, Phys. Rev. 133:A310 (1964).

42. D. G. Neal, Proc. Camb. Phil. Soc. 71:97 (1972).

43. B. P. Watson and P. L. Leath, Phys. Rev. B 9:4893 (1974).

44. P. L. Leath, Phys. Rev. B 14:5046 (1976).

45. M. F. Sykes, J. L. Martin, and J. W. Essam, J. Phys. A 6:1306 (1973).

46. M. F. Sykes, M. Glen, and D. S. Gaunt, J. Phys. A 7:L105 (1974).

47. E. Stoll and T. Schneider, Phys. Rev. A 6:429 (1972). 